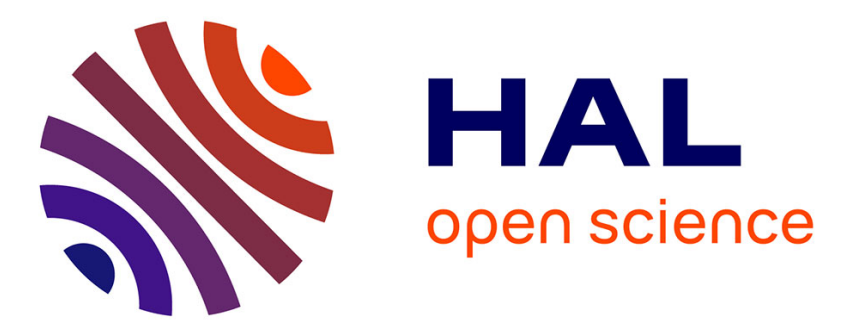

\title{
Effect of nonlinear superparamagnetic response on susceptibility curves for nanoparticle assemblies
}

Florent Tournus, Arnaud Hillion, Alexandre Tamion, Véronique Dupuis

\section{To cite this version:}

Florent Tournus, Arnaud Hillion, Alexandre Tamion, Véronique Dupuis. Effect of nonlinear superparamagnetic response on susceptibility curves for nanoparticle assemblies. Physical Review B: Condensed Matter and Materials Physics (1998-2015), 2013, 87 (17), pp.174404. 10.1103/PhysRevB.87.174404 . hal-03048691

\section{HAL Id: hal-03048691 \\ https://hal.science/hal-03048691}

Submitted on 15 Feb 2021

HAL is a multi-disciplinary open access archive for the deposit and dissemination of scientific research documents, whether they are published or not. The documents may come from teaching and research institutions in France or abroad, or from public or private research centers.
L'archive ouverte pluridisciplinaire HAL, est destinée au dépôt et à la diffusion de documents scientifiques de niveau recherche, publiés ou non, émanant des établissements d'enseignement et de recherche français ou étrangers, des laboratoires publics ou privés. 


\title{
Effect of nonlinear superparamagnetic response on susceptibility curves for nanoparticle assemblies
}

\author{
F. Tournus, * A. Hillion, A. Tamion, and V. Dupuis \\ Institut Lumière Matière, UMR5306 Université Lyon 1-CNRS, Université de Lyon 69622 Villeurbanne cedex, France
}

(Received 21 February 2013; revised manuscript received 11 April 2013; published 3 May 2013)

\begin{abstract}
We examine the effect of the applied magnetic field amplitude on zero field-cooled/field-cooled (ZFC/FC) curves, through the nonlinear susceptibility of superparamagnetic particles (i.e., at thermondynamic equilibrium, but taking into account the magnetic anisotropy). This nonlinear effect is shown to be the first to manifest itself when going away from the linear response regime (i.e., when the magnetic moment is simply proportional to the applied field), largely before the modification of the macrospin switching energy due to the external field. We demonstrate that it has a significant impact on ZFC/FC curves, especially for the low-temperature behavior of the FC curve, even in usual experimental conditions. We then show how this nonlinearity can be taken into account, in an easy way, to obtain a better modeling of the susceptibility curves and consequently a more reliable determination of the nanoparticles' magnetic properties. The theoretical considerations are confronted in a series of experimental measurements on Co nanoparticles.
\end{abstract}

DOI: 10.1103/PhysRevB.87.174404

PACS number(s): 75.75.-c, 75.50.Tt, 75.20.-g, 75.30.Gw

\section{INTRODUCTION}

The numerous potential applications of magnetic nanoparticles, in areas ranging from medicine to catalysis, are driving research on nanoparticle synthesis and characterization. ${ }^{1-6}$ In this context, low field susceptibility curves, following the so-called zero field-cooled/field-cooled (ZFC/FC) protocol, are widely used for the determination of the nanoparticle properties, in particular the distribution of magnetic anisotropy energy (MAE) and particle size.

This technique consists in measuring the magnetic moment of a nanoparticle assembly, under a low applied field (typically $50 \mathrm{Oe}$ ), as a function of temperature. The ZFC curve starts from low temperature (typically $2 \mathrm{~K}$ ), with a demagnetized sample cooled down from room temperature under no applied field. In contrast, for the FC curve the temperature is decreased from room to low temperature while the magnetic moment is measured, keeping the applied field constant. The obtained curves then reflect the progressive crossover, as a function of temperature $T$, between the blocked regime and the superparamagnetic (SP) regime (i.e., where the magnetic moments are at thermodynamic equilibrium). The main characteristic features of these susceptibility curves are the existence of a peak in the ZFC curve, occurring at a temperature $T_{\max }$, and the merging of the ZFC and FC curves when all the particles are in the superparamagnetic regime. The value of $T_{\max }$ is often used to characterize and compare, with a single figure, the magnetic anisotropy of nanoparticle samples. However, such a usage may be hazardous because both the magnetic anisotropy and the details of the size distribution control the shape of the ZFC/FC curves. ${ }^{7}$

In order to perform a more reliable analysis of these magnetization curves, a full modeling is yet possible both for the ZFC and the FC curve, using either a "two-states model" (or abrupt transition model, where the particles are supposed to be either blocked or SP), ${ }^{8-11}$ or a more elaborate "progressive crossover model.,"12,13 These two semianalytic models are equivalent as long as the MAE dispersion is large enough and with a carefully defined transition temperature (i.e., "blocking temperature"). ${ }^{7}$ One of the basic hypotheses of these models is that the applied magnetic field is low enough to observe a linear response of the nanoparticle assembly, which means that its magnetic moment (response) is simply proportional to the external field (i.e., the response scales linearly with the excitation). If we are in such a linear response regime, then the normalized $\mathrm{ZFC} / \mathrm{FC}$ curves measured with various field amplitudes $H$ should all be superimposed, as the total magnetic moment simply scales with $H$. However, this assumption is far from being systematically verified. Anyway, from an experimental point of view, a compromise needs to be found between having a fair signal-to-noise ratio and remaining close enough to the linear response regime.

On the other hand, it is well known that for applied magnetic fields of increasing amplitudes, the $T_{\max }$ value is shifting. ${ }^{14}$ The nonintuitive fact that this ZFC peak shift can be nonmonotonous has been discussed in the literature. ${ }^{15-17}$ In particular, Zheng et al. ${ }^{17}$ have pointed out that it can be explained by the contribution of SP particles with a size distribution. The influence of the applied field on the equilibrium magnetic moment is sometimes considered through a Langevin function, ${ }^{17-19}$ which is only justified at high temperature ${ }^{20,21}$ (i.e., far above the blocked-superparamagnetism crossover, when the anisotropy can be neglected).

In this paper, we examine the effect of the applied magnetic field amplitude on $\mathrm{ZFC} / \mathrm{FC}$ curves, through the nonlinear susceptibility of SP particles (i.e., at thermondynamic equilibrium, but taking into account the MAE). This nonlinear effect is shown to be the first to manifest itself when going away from the linear response regime, largely before the modification of the macrospin switching energy due to the external field. We demonstrate that it has a significant impact on $\mathrm{ZFC} / \mathrm{FC}$ curves, especially for the low-temperature behavior of the FC curve, even in usual experimental conditions. We then show how this nonlinearity can be taken into account, in an easy way, to obtain a better modeling of the susceptibility curves and consequently a more reliable determination of the nanoparticles' magnetic properties. The theoretical considerations are confronted in a series of experimental measurements on $\mathrm{Co}$ nanoparticles. 


\section{THEORETICAL ANALYSIS}

\section{A. Framework}

In the following, we make the usual assumption that magnetic nanoparticle samples can be described by independent macrospins of magnetic moment $\mu=M_{S} V$ and a uniaxial anisotropy corresponding to a MAE $K=K_{\text {eff }} V$. Here $M_{S}$ is the saturation magnetization, $V$ is the particle volume, and $K_{\text {eff }}$ is the effective anisotropy constant. We also assume that the easy magnetization axes of the nanoparticles are randomly oriented. The macrospin switching time is given by the Néel model: $\tau=\tau_{0} \exp \left(\frac{\Delta E}{k_{B} T}\right)$, where $\Delta E$ is the switching energy barrier, i.e., simply equal to $K$ in the absence of any external magnetic field. Moreover, the evolution of $K_{\text {eff }}, M_{S}$, and $\tau_{0}$ with temperature is neglected in the range of interest for ZFC/FC curves. It must be noted that the analysis presented in the following can still be applied to more complicated situations (texture of the macrospin orientations, dispersion of $K_{\text {eff }}$, evolution of $M_{S}, K_{\text {eff }}$, or $\tau_{0}$ with $T$ ), at the expense of a higher computational cost. The hypothesis of a macrospin behavior is crucial but is well verified for few-nanometerdiameter particles. ${ }^{22-24}$ From an experimental point of view, the most stringent hypothesis is certainly the absence of interparticle interactions, which requires particles far enough from each other (i.e., high dilution in the case of particles embedded in a nonmagnetic material). ${ }^{12,25-27}$

Before considering the realistic case of a nanoparticle assembly with a particle-size distribution, we discuss the case of a monodisperse assembly of randomly oriented macrospins. In a simplified model, which we call the "abrupt transition model," the macrospins of a given MAE are supposed to be either fully blocked (with a magnetic moment $m_{\mathrm{b}}$ ) or fully superparamagnetic, that is, at thermodynamic equilibrium (with a magnetic moment $m_{\mathrm{eq}}$ ). The expressions of $m_{\mathrm{b}}$ and $m_{\mathrm{eq}}$ are discussed further later. Then, the $\mathrm{ZFC}$ curve is given by $m_{\mathrm{ZFC}}=m_{\mathrm{b}}$ for $T<T_{B}$ and $m_{\mathrm{ZFC}}=m_{\mathrm{eq}}$ for $T \geqslant T_{B}$, where the blocking temperature $T_{B}$ establishes a transition between the two behaviors. The transition temperature corresponds to the situation when the measurement time $\tau_{m}$ (or effective measurement time, in the case of a continuous acquisition during a temperature sweep ${ }^{13,28}$ ) is equal to the switching time $\tau$, which means

$$
T_{B}=\frac{\Delta E}{k_{B} \ln \left(\tau_{m} / \tau_{0}\right)} .
$$

On the other hand, we have for the FC curve $m_{\mathrm{FC}}=m_{\mathrm{eq}}\left(T_{B}\right)$ for $T<T_{B}$ and $m_{\mathrm{FC}}=m_{\mathrm{eq}}$ for $T \geqslant T_{B}$.

One can distinguish the different effects of the applied magnetic field on the ZFC/FC curves (beyond the simple proportionality to the field, which does not modify the curves shape). The field amplitude $H$ will modify the macrospin switching time through a variation of $\tau_{0}{ }^{29}$ (this has only a small incidence) and more importantly through a change of $\Delta E$ in the exponential. ${ }^{14,30,31}$ In the end this will affect the blocking temperature of particles of a given MAE. Besides, the equilibrium (SP) magnetic moment $m_{\mathrm{eq}}$ also depends on $H$ and the deviation from the linear behavior $m_{\mathrm{eq}}$, lin $=$ $\mu_{0} \mu^{2} H /\left(3 k_{B} T\right)$ will contribute to modify the $\mathrm{ZFC} / \mathrm{FC}$ curves shape when the applied field is increased. Interestingly, it should be noted that the blocked moment $m_{\mathrm{b}}$ is strictly linear in $H$ (because it is related to the transverse susceptibility ${ }^{13}$ ), as long as the saturation is not reached:

$$
m_{\mathrm{b}}=\frac{\mu_{0} \mu^{2} H}{3 K} \text {. }
$$

While the magnetic field effect on $\Delta E$ and even $\tau_{0}$ has already been considered in previous studies, ${ }^{14,16,17,30,32}$ the dependence of $m_{\mathrm{eq}}$ upon $H$ has been under-rated. Let us emphasize that $m_{\mathrm{eq}}(H)$ does not correspond to a simple Langevin function. Indeed, the Langevin function is the thermodynamic equilibrium response of a particle assembly only in the case of a vanishing magnetic anisotropy. ${ }^{20,21,28}$ This is a crucial point: The particles' anisotropy still plays a role in the SP contribution, especially close to $T_{B}$. In particular, we can expect it to have a strong impact on the FC curve, because $m_{\text {eq }}\left(T_{B}\right)$ is involved (see above).

These observations also hold for the more elaborate progressive crossover model, where we can write ${ }^{13}$

$$
m_{\mathrm{ZFC}}=m_{\mathrm{b}} e^{-\delta t / \tau}+m_{\mathrm{eq}}\left(1-e^{-\delta t / \tau}\right)
$$

and

$$
m_{\mathrm{FC}}=m_{\mathrm{eq}}\left(T_{B}\right) e^{-\delta t / \tau}+m_{\mathrm{eq}}\left(1-e^{-\delta t / \tau}\right),
$$

$\delta t$ being an effective waiting time related to the temperature sweeping rate $r_{T}$. As explained in Ref. 13, $\delta t$ depends on the anisotropy and on the temperature $[\delta t \simeq$ $\left.0.6727\left(T / r_{T}\right)\left(k_{B} T / K\right)^{0.9}\right]$, which means that it varies among the particles and along the $\mathrm{ZFC} / \mathrm{FC}$ curves. In this case, we have (for a negligible applied field) ${ }^{13}$

$$
T_{B} \simeq \frac{K / k_{B}}{0.9283 \ln \left(\frac{K}{k_{B} \tau_{0} r_{T}}\right)-3.69} .
$$

To go further, we can point out that the effects of the applied field $H$ on the macrospin switching time are controlled by the dimensionless parameter $h=H / H_{A}$, where $H_{A}$ is the anisotropy field defined by $H_{A}=2 K_{\text {eff }} /\left(\mu_{0} M_{S}\right)$. These effects are then negligible as long as $h \ll 1$. On the other hand, the deviation of $m_{\mathrm{eq}}$ from a linear response to the applied magnetic field is controlled by another dimensionless parameter (ratio between Zeeman and thermal energies) ${ }^{33}$ :

$$
\xi=\frac{\mu_{0} M_{S} V H}{k_{B} T} .
$$

Therefore, the linear approximation for $m_{\mathrm{eq}}$ is acceptable as long as $\xi \ll 1$, where we can write $m_{\text {eq }} \simeq m_{\text {eq, lin }}$. By introducing a third dimensionless parameter $\sigma=K_{\text {eff }} V /\left(k_{B} T\right)$, which reflects the relative importance of anisotropy vs thermal effects, we can see how $h$ and $\xi$ are related: $\xi=2 \sigma h$. The SP regime (equilibrium response) takes place typically for $\sigma \leqslant 25$ [this comes from the fact that for $\mathrm{ZFC} / \mathrm{FC}$ measurements we have $\left.\ln \left(\tau_{m} / \tau_{0}\right) \simeq 25\right]$. This means that around $T_{B}$, at the onset of the SP regime, we can write $\xi \simeq 50 h$. For instance, we can have a situation where $h=0.01$, and hence $T_{B}$ will not be noticeably changed, while $\xi \simeq 0.5$ around $T_{B}$ so that the SP contribution will clearly deviate from $m_{\mathrm{eq}, \mathrm{lin}}$. This demonstrates that the applied field amplitude $H$ modifies $m_{\text {eq }}$ much before having a sizable effect on the blocking temperature (through the macrospin switching time $\tau$ ).

As a consequence, there exists an intermediate regime where $h \ll 1$ but $\xi$ is no more negligible, and hence 
the nonlinear effects in $m_{\mathrm{eq}}$ start to be significant (i.e., we are facing a nonlinear superparamagnetic response) while the macrospin switching is almost unaffected by $H$. In the following, we consider this particular regime, which is the first to appear when one deviates from the linear response regime (at low field).

\section{B. Third-order magnetic susceptibility and ZFC/FC simulation}

We consider a system of randomly oriented uniaxial macrospins, having the same volume $V$, and consequently the same magnetic moment $\mu=M_{S} V$ and MAE $K=K_{\text {eff }} V$. We can determine its equilibrium response (component along the applied field direction, $m_{\text {eq }}$ ) to an external field $H$, at a temperature $T$. This can be done numerically without any difficulty, as reported in several studies, ${ }^{20,21,33,34}$ using statistical physics formulations involving the system partition function.

Then, a Taylor expansion can be made as a function of $\xi$ in reduced units. One can write

$$
m_{\mathrm{eq}} / m_{S}=\frac{\xi}{3}+\alpha \xi^{3}+\cdots,
$$

where $m_{S}$ is the sample saturation magnetic moment. Thus, the first nonlinear term is proportional to the reduced third-order susceptibility $\alpha$. This coefficient depends only on the parameter $\sigma$. In the limit of small $\sigma$ or large $\sigma$ values, $\alpha(\sigma)$ can be expressed analytically using power series of $\sigma$ or $1 / \sigma$. We can write $^{33}$

$$
\alpha=-\frac{1}{45}-\frac{8}{10125} \sigma^{2}+\cdots \quad \text { for } \quad \sigma \ll 1
$$

and

$$
\alpha=-\frac{1}{15}+\frac{2}{15 \sigma}+\cdots \text { for } \sigma \gg 1 .
$$

The extreme cases correspond to a Langevin function ( $\sigma=$ 0 , no anisotropy barrier) and to a Ising function $(\sigma \rightarrow \infty$, only two possible orientations) with respectively $\alpha=-1 / 45$ and $\alpha=-1 / 15$. In the case relevant for $\mathrm{ZFC} / \mathrm{FC}$ measurements, we are mostly interested in knowing the $\alpha$ value for $\sigma$ around 20-25. Let us insist on the fact that in this range $m_{\text {eq }}$ is closer to a Ising function than to a Langevin function. Note that limiting the nonlinear expansion of $m_{\mathrm{eq}}$ to the third-order susceptibility is a good approximation up to $\xi \simeq 0.5-1$, which corresponds to an applied field $H / H_{A} \simeq 0.01-0.02$. Figure 1 indeed displays the error on $m_{\text {eq }}$ induced by such a truncation to the third-order susceptibility. It also compares this truncation to the linear approximation and a Langevin function. Note also that because $\xi$ is inversely proportional to $T$, the truncation becomes a better approximation when going away from the blocking temperature. Beyond the fact that $m_{\text {eq }}$ does not correspond to a Langevin function (see Fig. 1), taking into account the true third-order magnetic susceptibility constitutes an essential improvement because the dependence of $\alpha$ on $\sigma$ (i.e., on the particle anisotropy and size) is then considered. This will be very important for a correct description of the $\mathrm{ZFC} / \mathrm{FC}$ curves in the case of a particle-size distribution.

In order to efficiently incorporate this third-order susceptibility effect in $\mathrm{ZFC} / \mathrm{FC}$ simulations, one needs an analytical expression of $\alpha(\sigma)$ in the entire range of interest for SP

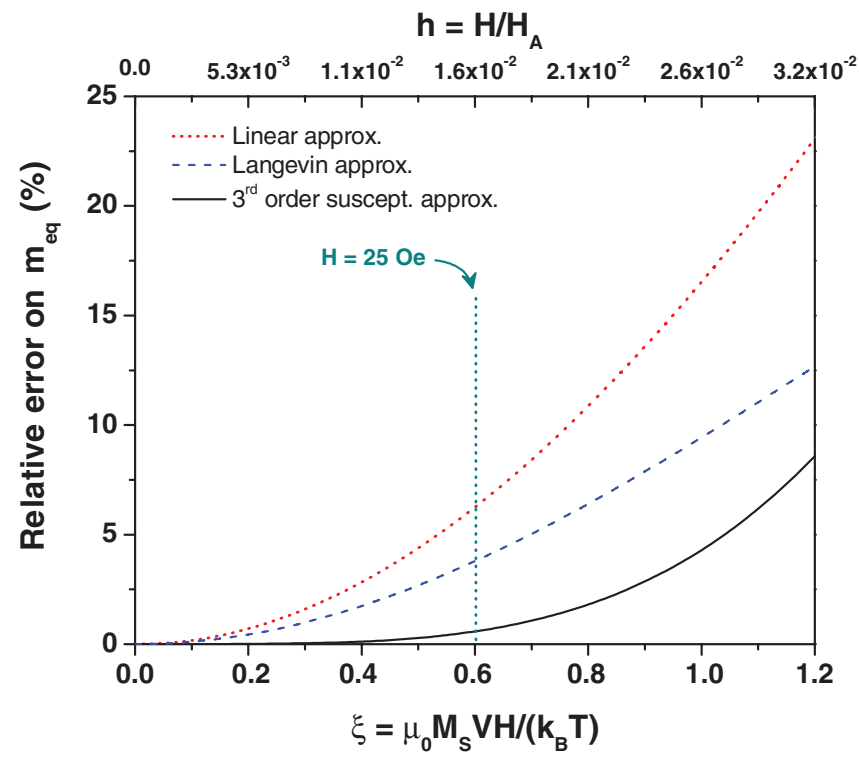

FIG. 1. (Color online) Relative error on the equilibrium magnetic moment $m_{\text {eq }}$, as a function of the dimensionless parameter $\xi$, for different approximations: the linear response approximation (red [gray] dotted line), the Langevin approximation (blue [gray] dashed line), and the true nonlinear response truncated to the third-order susceptibility (black solid line). The calculations are done for particles of 5 -nm diameter, with $M_{S}=1.35 \times 10^{6} \mathrm{~A} / \mathrm{m}, K_{\text {eff }}=$ $100 \mathrm{~kJ} / \mathrm{m}^{3}$ (which corresponds to $T_{B} \simeq 18 \mathrm{~K}$ and $H_{A} \simeq 1500 \mathrm{Oe}$ ), at a temperature of $25 \mathrm{~K}$ (i.e., $\sigma \simeq 19$ ).

particles (i.e., from $\sigma=0$ to about 25). Because we can already describe with high precision the two extreme regimes $(\sigma \ll 1$ and $\sigma \gg 1)$, we propose a linear interpolation ${ }^{35}$ between the values $\alpha\left(\sigma_{\min }\right)$ and $\alpha\left(\sigma_{\max }\right)$, where $\sigma_{\min }$ and $\sigma_{\max }$ are the boundary values where the extreme regimes approximations start to fail. As shown in Fig. 2, one can find very good polynomial fits in either $\sigma$ or $1 / \sigma$ (these fits are slightly different from the Taylor expansions but allow us to extend the domain of applicability), up to $\sigma \simeq 2$ on the low- $\sigma$ side and down to $\sigma \simeq 5.5$ on the high- $\sigma$ side: The linear interpolation is then limited to $\sigma \in[2,5.5]$ and appears very satisfactory.

We now turn to ZFC/FC simulations, incorporating the applied field effect on $m_{\text {eq }}$ through the analytical expression of the reduced third-order susceptibility discussed above. As already mentioned, two models can be used to describe the evolution from the blocked regime to the SP regime: ${ }^{13}$ The abrupt transition model (ATM), where there is a discontinuity for the ZFC at the transition temperature (in a case of a single MAE), or the progressive crossover model (PCM), where the evolution between the two regimes is gradual, resulting in continuous $\mathrm{ZFC}$ curves even for a single MAE. In both formulations (see above), the equilibrium magnetic moment $m_{\mathrm{eq}}$ appears in a very transparent way, so that there is no difficulty to add the third-order susceptibility contribution (analytical approximation) to the usual linear response $m_{\text {eq,lin }}=\mu_{0} \mu^{2} H /\left(3 k_{B} T\right)$.

Figure 3(a) shows the effect of the applied field amplitude on the $\mathrm{ZFC} / \mathrm{FC}$ curves, for a single MAE (corresponding typically to Co clusters in the fcc phase with a 5-nm diameter, under a 25-Oe magnetic field). Different approximations for 
(a)

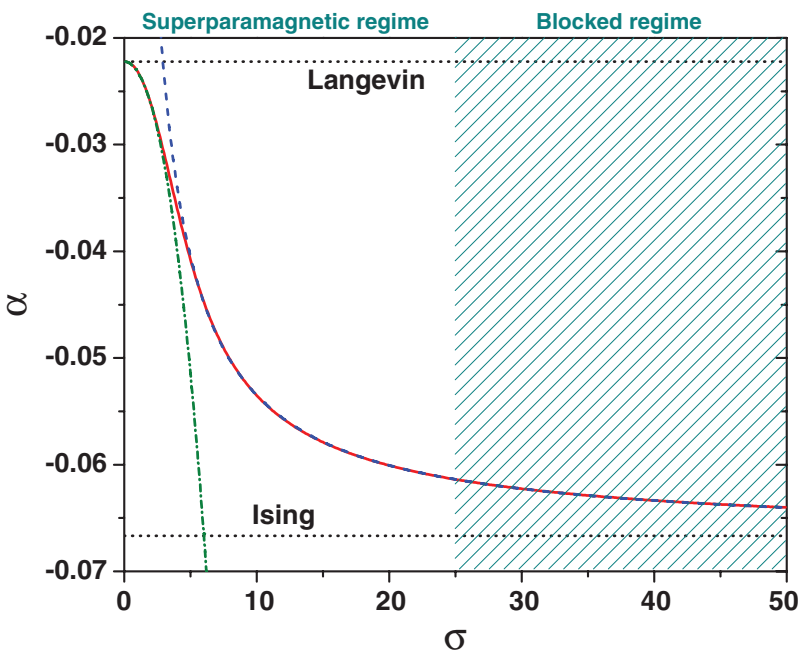

(b)

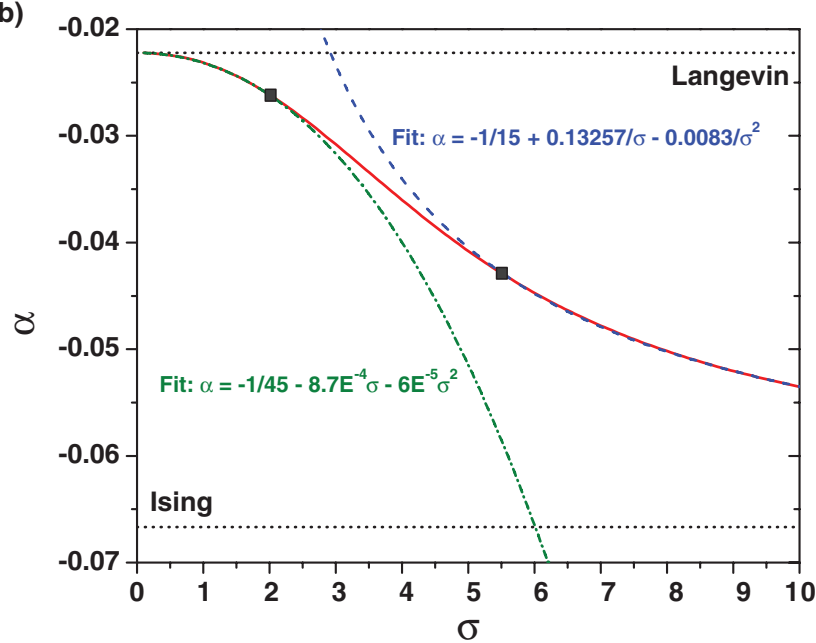

FIG. 2. (Color online) (a) Numerical calculation of the reduced third-order susceptibility $\alpha$ (red [gray] solid line) as a function of $\sigma$, compared to the low- $\sigma$ and high- $\sigma$ analytical approximations (blue [gray] dashed line and green [gray] dash-dotted line). The extreme cases, corresponding to a Langevin or a Ising function, are indicated with horizontal dotted lines. In a ZFC/FC measurement, the superparamagnetic regime (i.e., thermodynamic equilibrium) is observed typically for $\sigma \leqslant 25$, whereas the region $\sigma>25$ (hatched region) corresponds to the blocked regime. (b) Closer view, for $\sigma$ between 0 and 10, of the numerically determined $\alpha(\sigma)$ value (red [gray] solid line) and the low- $\sigma$ and high- $\sigma$ analytical approximations (blue [gray] dashed line and green [gray] dash-dotted line). The two black squares indicate the $\sigma_{\min }$ and $\sigma_{\max }$ values and materialize the range where a linear interpolation will be used for $\alpha(\sigma)$.

the equilibrium response are compared (see the Appendix for the analytical expression of the curves for each approximation): The linear approximation, the Langevin approximation, and the third-order susceptibility approximation. In this case, in addition to simulations using the PCM, what would be obtained for the more common ATM is also displayed. Note that the illustrative case considered here corresponds to $h \simeq 0.017$, a blocking temperature $T_{B} \simeq 18 \mathrm{~K}$, a $\sigma$ value around 26 at $T_{B}$, and $\xi \simeq 0.9$ at $T_{B}$. We are thus typically in the regime described previously, where the nonlinearity cannot be neglected while the energy barrier (and consequently $T_{B}$ ) is almost unaffected ( $h \ll 1$ is still satisfied). One can immediately see that there is a clear deviation from the linear case (i.e., what would be obtained with a very low applied field), especially around the ZFC peak. As far as the FC curve is concerned, the most striking effect is the significant decrease of (a)

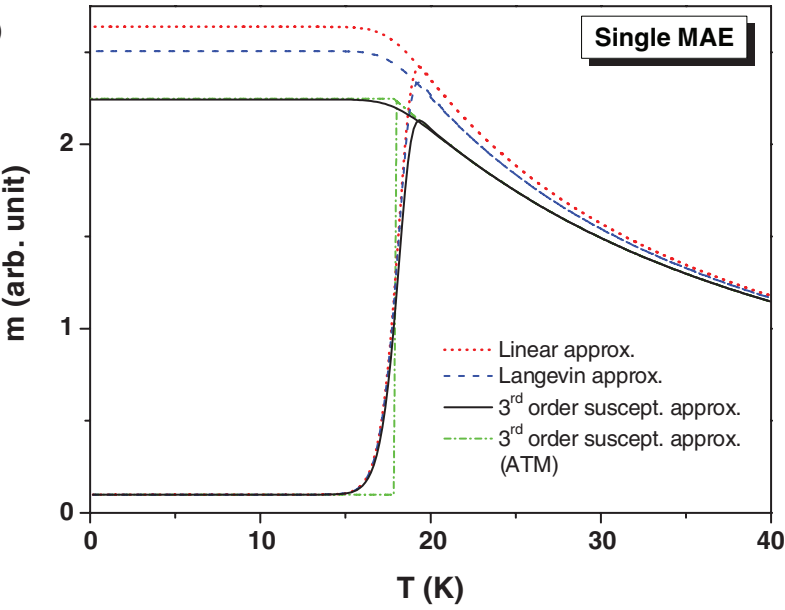

(b)

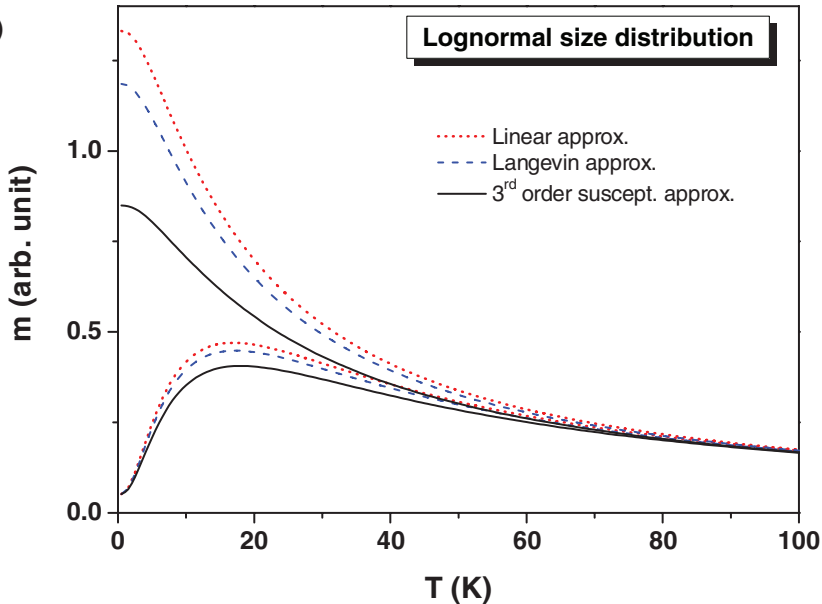

FIG. 3. (Color online) ZFC/FC curves simulations using the progressive crossover model (PCM), for a single MAE (a) or for a MAE distribution due to a log-normal particle-size distribution (b). Different approximations for $m_{\mathrm{eq}}$ are compared (the analytical expressions are summarized in the Appendix): the linear response approximation (red [gray] dotted line), the Langevin approximation (blue [gray] dashed line), and the true nonlinear response truncated to the third-order susceptibility (black solid line). The calculations are done for particles with $M_{S}=1.35 \times 10^{6} \mathrm{~A} / \mathrm{m}, K_{\text {eff }}=100 \mathrm{~kJ} / \mathrm{m}^{3}$ (which corresponds to $H_{A} \simeq 1500$ Oe), with a 25 -Oe applied magnetic field (i.e., $h \simeq 0.017$ ). $\tau_{0}$ is fixed to $10^{-10} \mathrm{~s}$ and the temperature sweeping rate is $r_{T}=0.033 \mathrm{~K} / \mathrm{s}$. The particle diameter is equal to $5 \mathrm{~nm}$ (single MAE case, case a), while it follows a log-normal distribution (case b) with a median diameter of $3 \mathrm{~nm}$ and a dispersion parameter of 0.3. For the single MAE case, the curves corresponding to the abrupt transition model (ATM, dash-dotted line) are also shown. In the case of a significant MAE distribution (as in case b) the ATM and PCM are equivalent. 
(a)

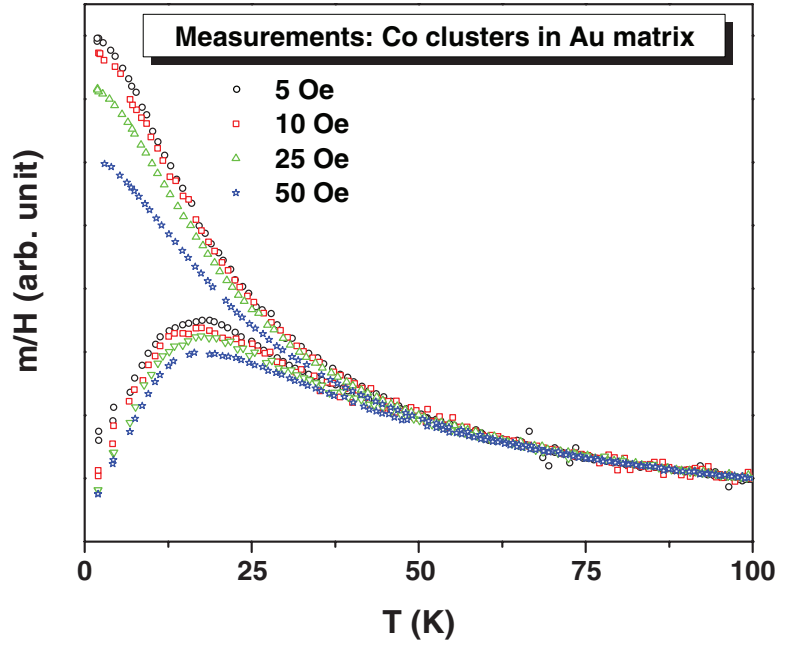

(b)

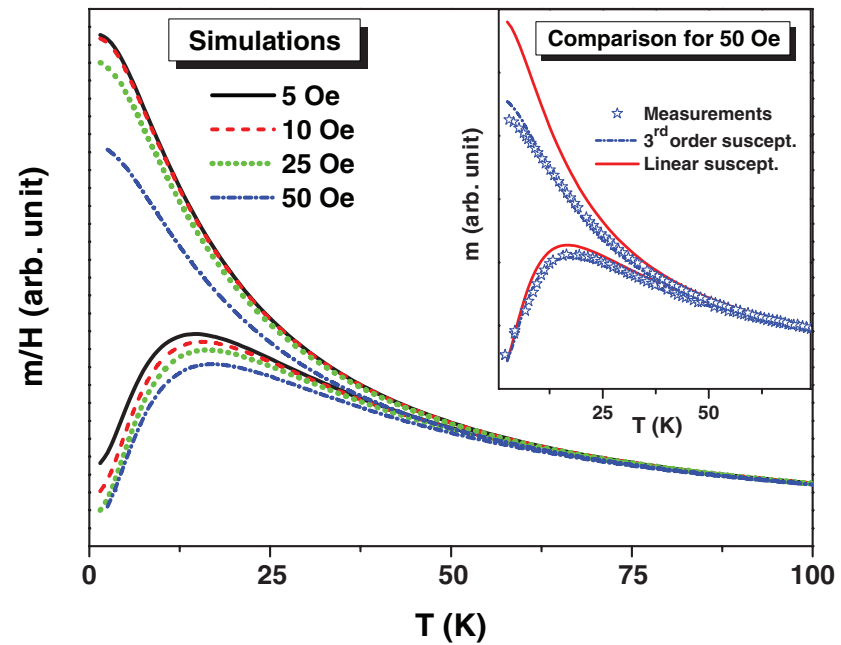

FIG. 4. (Color online) Normalized ZFC/FC curves measured for a sample of Co nanoparticles embedded in a gold matrix, with different applied field amplitudes (a). Simulated ZFC/FC curves, taking into account the third-order susceptibility in the superparamagnetic contribution, for different applied magnetic fields (b). In insert, the experimental curves with a 50-Oe applied field are compared to the curves simulated with the third-order susceptibility approximation and the linear response approximation. The nanoparticles have a log-normal size distribution (median diameter of $2.75 \mathrm{~nm}$ and dispersion parameter of 0.26 ) and a $K_{\text {eff }}=162 \mathrm{~kJ} / \mathrm{m}^{3}$. A residual field of $0.7-O e$ is included.

the low temperature limit, which can easily be explained by the fact that the third-order susceptibility is always negative (i.e., it decreases the response magnitude as compared to the linear regime). The inability of a Langevin description to correctly account for the applied field effects is also clearly visible. In Fig. 3(b), the same comparison between the different approximations (linear response, Langevin function, and response including the third-order susceptibility) for the superparamagnetic particles response is done for the more realistic case of a particle-size distribution (log-normal in this case). The same type of behavior as for a single particle size is observed: The FC curve slope is significantly decreased, and the ZFC peak has a reduced amplitude. Note that the beginning of the ZFC (low temperature) remains unchanged (because of $m_{\mathrm{b}}$ linearity), as well as the high-temperature limit, where both $\sigma$ and $\xi$ go to zero so that the system's response is simply given by the linear susceptibility of a paramagnet (i.e., $1 / T$ evolution).

This theoretical analysis demonstrates that the nonlinearity of the SP contribution must be considered in order to accurately analyze experimental curves. Using a Langevin function does not at all constitute a reliable solution. Of course, for applied field amplitudes that are too large, considering only the thirdorder susceptibility is not enough and the simple approach presented here cannot be applied (moreover, in this case the blocking temperatures are also significantly affected by the magnetic field). From an experimental point of view, however, there is no real interest in choosing such conditions, highly deviating from the linear response regime.

\section{EXPERIMENTAL STUDY}

The evolution of the ZFC/FC curves' shapes, when varying the applied magnetic field amplitude, has been experimentally studied on a Co nanoparticle assembly. The sample consists of Co nanoparticles (around $3 \mathrm{~nm}$ in diameter, with a lognormal size distribution) synthesized by laser vaporization and deposited in ultra-high-vacuum conditions using low- energy cluster beam deposition (LECBD). ${ }^{36,37}$ During particle deposition, a gold matrix is codeposited (by electron beam evaporation) so that the magnetic clusters are embedded in $\mathrm{Au}$ with a high dilution ( $0.5 \%$ in volume), resulting in negligible interparticle interactions. ${ }^{26}$

ZFC/FC measurements have been performed with different applied field amplitudes, using a superconducting quantum interference device (SQUID) magnetometer (Quantum Design MPMS 5XL). As can be seen in Fig. 4(a), there is a striking evolution of the curves' shapes (normalized by the applied field) with a flattening of the FC curve (low-temperature limit) and a decrease of the ZFC peak amplitude when the magnetic field is increased from 5 to $50 \mathrm{Oe}$. A first observation is that, as far as the FC curve is concerned, the linear response (invariance of the curves shape) is satisfied up to a 10-Oe applied field in the present case. The fact that $\mathrm{ZFC}$ curves are not superimposed, as is the case for FC curves (5- and 10-Oe curves, and it almost holds for the 25-Oe FC too), may seem surprising. Indeed, let us remember that the blocked contribution is not affected by the applied field (simple linear behavior) so that when all the particles are blocked (which is almost the case at the lowest temperature of $2 \mathrm{~K}$ ) the normalized ZFC curves should have the same value.

The downshift of the ZFC at low temperature, when the field is increased, can fully be explained by the existence of a small residual field in the SQUID when cooling the sample in supposedly "zero field-cooled" conditions. As it has been discussed previously, a tiny residual field of the order of $1 / 25$ (i.e., 4\%) of the measurement field amplitude is enough to perturb the low-temperature part of a ZFC curve. ${ }^{13}$ The effect of the residual field $H_{\text {res }}$ can be taken into account in the simulations: We first compute the low-temperature limit of a FC curve with $H_{\text {res }}$ as the applied field and then use this value as the starting point of the experimental ZFC curve. Here, we find that the measurements are consistent with a $0.7-O e$ residual field, which would be fully negligible for many measurements but has a detectable impact in the case of ZFC curves acquired 
with a small amplitude field: Such a $H_{\text {res }}$ represents around $14 \%$ of the measurement field in the case of $H=5$ Oe and $7 \%$ for $H=10$ Oe. When the applied field becomes large enough, this residual field has a negligible effect on the ZFC starting point, which is precisely what we observe for $25-\mathrm{Oe}$ and 50-Oe measurements.

The other features (flattening of the FC curve and decrease of the ZFC peak) are well reproduced by the simulations [see Fig. 4(b)], using the framework exposed above, which includes the third-order susceptibility in the equilibrium magnetic response. These effects then result only from the nonlinearity of $m_{\text {eq }}$. Interestingly, we have found that it is often possible to fit $\mathrm{ZFC/FC}$ curves, even in the nonlinear regime discussed here, with the simple linear response formulation. As has just been demonstrated above, such a successful fit does not prove in any way that the magnetic response to the external field is indeed linear. The series of experimental curves [Fig. 4(a)] can be fitted using the previously reported "triple-fit" method (simultaneous fit of the 300-K superparamagnetic $m(H)$ curve and $\mathrm{ZFC} / \mathrm{FC}$ susceptibility curves). ${ }^{12}$ The FC flattening is in fact not prohibitive for the fit using linear expressions, because the low- $T$ limit of the FC is treated as an adjustable parameter (it is chosen to give the experimental value). However, depending on which measurement field is considered, the parameters obtained by a best-fit procedure are not exactly identical. While the impact on the deduced particle-size distribution is very limited (0.1-nm change in the median diameter and 0.01 variation in the log-normal dispersion), the anisotropy constant increases from $K_{\text {eff }}=162 \mathrm{~kJ} / \mathrm{m}^{3}$ for $H=5$ Oe to $K_{\text {eff }}=178 \mathrm{~kJ} / \mathrm{m}^{3}$ for $H=50$ Oe. In contrast, a fit with the third-order nonlinear susceptibility provides a constant $K_{\text {eff }}=162 \mathrm{~kJ} / \mathrm{m}^{3}$ value. In the case of a 50 -Oe measurement, and for this particular sample, neglecting the nonlinearity of $m_{\mathrm{eq}}$ would result in a $10 \%$ overestimation of the magnetic anisotropy constant. The inset in Fig. 4(b) displays the best fit of the experimental curves with the third-order susceptibility approximation and shows how important the nonlinear effects are (the linear susceptibility approximation gives drastically different curves).

Note that neglecting the effect of the residual field for low-field measurements is worse and can easily lead to a $20 \%$ error on $K_{\text {eff }}$, which is then underestimated. More generally, taking into account the nonlinearity effect allows one to avoid the potentially significant error (even when remaining in the regime where $h \ll 1$ ) on the magnetic anisotropy constant that would be inferred from a fit based on a linear response model. For instance, including the third-order susceptibility would avoid a $18 \%$ overestimation in the case displayed in Fig. 3(b), while in other cases errors up to $30 \%$ on $K_{\text {eff }}$ can be avoided. A nonlinear modeling is then clearly beneficial for the accuracy of the magnetic parameters determined from experimental curves. It is also important to note that a slight shift of the ZFC curve maximum temperature $T_{\max }$ can be observed as a function of the applied field, even if the macrospin switching energy barriers are not affected (i.e., $h$ remains very small). Such a shift can be obtained with a particle-size distribution, simply because there exists a nonlinearity in $m_{\mathrm{eq}}$, and may be amplified by the existence of a tiny residual field in the magnetometer during the zero fieldcooling step.

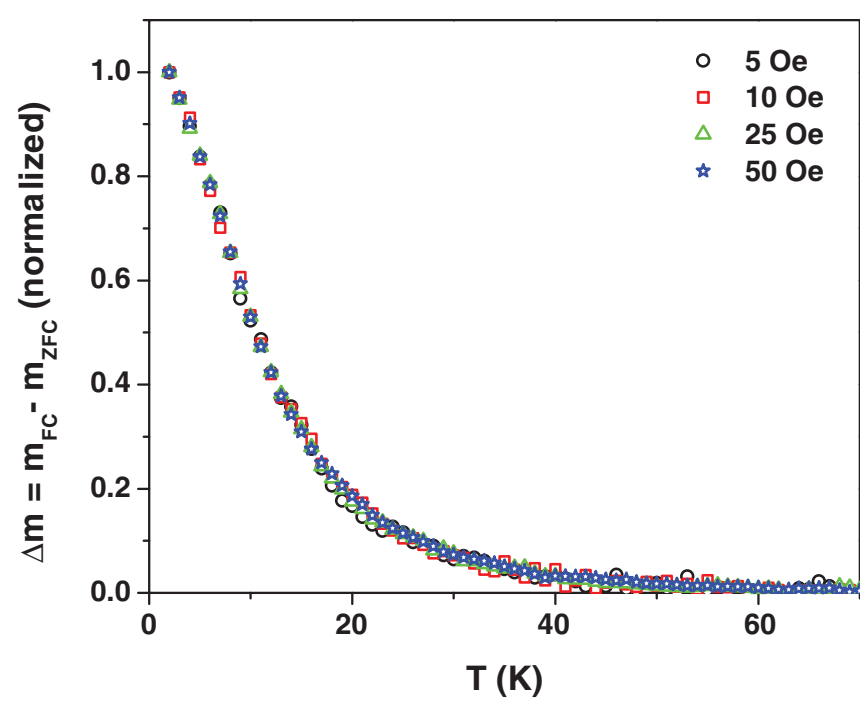

FIG. 5. (Color online) Experimental normalized $\Delta m=m_{\mathrm{FC}}-$ $m_{\mathrm{ZFC}}$ curves for the sample of Co nanoparticles embedded in a gold matrix, with the different applied field amplitudes.

According to the ZFC/FC curve expressions, ${ }^{7,13}$ both the effect of a residual field and of a nonlinearity in $m_{\text {eq }}$ should vanish when considering the difference $\Delta m=m_{\mathrm{FC}}-m_{\mathrm{ZFC}}$. As expected, in sharp contrast with the change in the ZFC/FC curves with the applied field amplitude, the normalized $\Delta m$ curves are found to be almost identical (see Fig. 5). These curves are the signature of the gradual unblocking of the particles with increasing temperature: They are similar to remanence $m_{R}(T)$ measurements and only bear the signature of the blocked nanoparticles. $\Delta m$ is also directly related to the distribution of blocking temperatures in the nanoparticle assembly, ${ }^{7}$ and thus it reflects the switching energy distribution in the sample. The fact that in the present experimental case $\Delta m$ does not evolve with the applied field, in the range considered here, is a proof that we are still in the $h \ll 1$ regime where the field effect on the energy barriers can be neglected. This justifies the use of the framework exposed above where only the equilibrium contribution is modified by $H$.

One may want to take advantage of these properties of the $\Delta m=m_{\mathrm{FC}}-m_{\mathrm{ZFC}}$ curve, namely the insensitivity to a residual field and to a nonlinearity in $m_{\text {eq }}$. Indeed, as already evoked in a previous work, ${ }^{7} \Delta m(T)$ can be fitted directly using a semianalytical model. In the case of a particle-size distribution function $\rho(V)$, one can write for the progressive crossover model

$$
\Delta m \propto \frac{\mu_{0} M_{S}^{2} H}{3 K_{\text {eff }}} \int_{0}^{\infty} V e^{-\delta t / \tau} \rho(V) d V
$$

where both $\tau$ and $\delta t$ (to a much lesser extent) depend on $T$ and $V$. Similarly, with the abrupt transition model, one can write

$$
\Delta m \propto \frac{\mu_{0} M_{S}^{2} H}{3 K_{\text {eff }}} \int_{V_{\text {lim }}}^{\infty} V \rho(V) d V,
$$

with $\quad V_{\lim }(T)=\ln \left(\tau_{m} / \tau_{0}\right) k_{B} T / K_{\text {eff }}$, which corresponds typically to $V_{\lim } \simeq 25 k_{B} T / K_{\text {eff }}$. There is thus no difficulty 
to fit an experimental $\Delta m$ curve, which can give access to the particle-size distribution and the anisotropy constant. However, going from two curves (FC and $\mathrm{ZFC}$ ) to only one results in a loss of information (contribution of the equilibrium magnetic moments) and transforms a highly discriminating feature (the ZFC peak) into a smooth curve, which appears to be less sensitive to a parameter variation. In the end, we believe that directly analyzing ZFC/FC curves is preferable.

Finally, this study stresses that special attention must be paid to the choice of the applied field for ZFC/FC susceptibility measurements. A compromise has to be found between a high signal (and consequently a better quality of the experimental curves) and a strong deviation from linearity, which precludes a simple theoretical modeling of the curves. It is then strongly advisable to check in which regime we are (linear response, moderate nonlinear response with $h \ll 1$, highly nonlinear response) when analyzing a particular sample. Some conditions may be suited for one case and not for another one: In particular, according to the material considered, the anisotropy field $H_{A}$ can vary a lot, thus changing substantially the value of $h$ and the parameter $\xi$ around the blocking temperature. It must be kept in mind that with a fixed acquisition magnetic field, the nonlinearity effects will be more pronounced for systems with a small $H_{A}$. By evaluating the maximum value of $\xi$ corresponding to a given experimental situation, one can ensure that a reliable modeling of the curves is then possible.

\section{CONCLUSION}

We have shown how the applied magnetic field amplitude can alter the shape of ZFC/FC susceptibility curves of a nanoparticle assembly. The first effect, when deviating from the linear response regime valid at very low acquisition field, is to modify the magnetic behavior of superparamagnetic particles: A nonlinearity appears in the equilibrium magnetic moment $m_{\text {eq }}$, the third-order susceptibility being the first component that needs to be considered. Such a nonlinear effect can be significant while the impact of the applied magnetic field on the switching energy distribution (and hence on the particles blocking temperatures) can safely be neglected.

In this moderate nonlinear regime, a simple theoretical modeling of this nonlinear effect is possible with an accurate analytical approximation of the reduced third-order equilibrium susceptibility. This framework is then fully compatible with the usual theoretical description of $\mathrm{ZFC} / \mathrm{FC}$ curves (ATM or PCM) and only involves a marginal additional computational cost. Unfortunately, a simple and reliable theoretical description seems impossible for large applied field amplitudes, where the third-order susceptibility is not sufficient and where the switching energy barrier are also affected.

The most visible effect of the appearance of a $m_{\text {eq }}$ nonlinearity is the flattening of the FC curve (lowering of the low-temperature limit). In the case of a particle-size distribution, a slight shift of the ZFC peak temperature with the field is also possible, even if the individual particle blocking temperatures remain unchanged. It must be noted that the use of a Langevin function to describe the field dependence of the superparamagnetic contribution is not physically sound, as it misses the dependence of the nonlinear susceptibility on the parameter $\sigma=K_{\text {eff }} V /\left(k_{B} T\right)$. As long as the applied field is not too high, taking into account the nonlinear behavior of the equilibrium magnetic response enables a more accurate determination of the nanoparticles' magnetic properties from the fit of experimental ZFC/FC curves. This has been illustrated on a sample of Co nanoparticles embedded in a gold matrix, characterized with an applied magnetic field ranging from 5 to $50 \mathrm{Oe}$. In this case, the linear response regime is only met at low acquisition fields ( $H \leqslant 10$ Oe).

Experimentally, when choosing the applied field amplitude for $\mathrm{ZFC} / \mathrm{FC}$ measurements, it is advisable to find a good compromise between having an intense signal and keeping a moderate nonlinearity in the nanoparticles' magnetic response. The existence of a very small residual field in the magnetometer (even less than $1 \mathrm{Oe}$ ), during the zero field-cooling step, has been shown to be critical in the case of low acquisition field amplitudes (for instance, 5 or $10 \mathrm{Oe}$ ). With these considerations, we think that in many cases the most judicious choice is to use a 25-Oe applied field for ZFC/FC measurements, keeping in mind that the conditions of validity of the different approximations $(h \ll 1$, small $\xi$ ) vary with the experimental situation considered (in particular with the material). Interestingly, and as expected from the theoretical description, the quantity $\Delta m=m_{\mathrm{FC}}-m_{\mathrm{ZFC}}$ appears to be insensitive to the nonlinearity in the superparamagnetic response and to the effect of a residual field. Since it can also be easily modeled, this curve which reflects the unblocking of the particles as a function of temperature may be useful in some cases.

Finally, the flattening of the FC curve with increasing field amplitude is reminiscent of the effect of interparticle interactions. This should be related to the existence, in concentrated samples, of an internal effective field reflecting the dipolar interactions among the nanomagnets.

\section{ACKNOWLEDGMENTS}

The authors acknowledge D. Ferrah and E. Bonet for fruitful discussions, the PLYRA for nanoparticle sample preparation, and the CML for SQUID magnetometry measurements.

\section{APPENDIX: SUMMARY OF THE ANALYTICAL EXPRESSIONS USED FOR ZFC/FC MODELLING}

In the present article, analytical expressions have been used to describe the evolution of the magnetic moment, in a ZFC/FC measurement, for an assembly of particles having the same volume $V$ and consequently the same anisotropy $K=K_{\text {eff }} V$ and magnetic moment $\mu=M_{S} V$. For a particle assembly with a size distribution, these analytical expressions are numerically integrated over the particle volume.

The progressive crossover model described in Ref. 13 allows us to write

$$
m_{\mathrm{ZFC}}=m_{\mathrm{b}} e^{-\delta t / \tau}+m_{\mathrm{eq}}\left(1-e^{-\delta t / \tau}\right)
$$

and

$$
m_{\mathrm{FC}}=m_{\mathrm{eq}}\left(T_{B}\right) e^{-\delta t / \tau}+m_{\mathrm{eq}}\left(1-e^{-\delta t / \tau}\right)
$$


with

$$
\begin{aligned}
m_{\mathrm{b}} & =\frac{\mu_{0} \mu^{2} H}{3 K}, \quad T_{B}=\frac{K / k_{B}}{0.9283 \ln \left(\frac{K}{k_{B} \tau_{0} r_{T}}\right)-3.69}, \\
\delta t & =0.6727 \frac{T}{r_{T}}\left(\frac{k_{B} T}{K}\right)^{0.9}, \quad \text { and } \tau=\tau_{0} \exp \left(\frac{K}{k_{B} T}\right) .
\end{aligned}
$$

Different approximations can then be used for the equilibrium magnetic moment $m_{\text {eq }}$.

(1) Linear response approximation:

$$
m_{\mathrm{eq}}=\frac{\mu_{0} \mu^{2} H}{3 k_{B} T} .
$$

(2) Langevin approximation:

$$
m_{\mathrm{eq}}=\mu\left[\operatorname{coth}\left(\frac{\mu_{0} \mu H}{k_{B} T}\right)-\frac{k_{B} T}{\mu_{0} \mu H}\right] .
$$

(3) Third-order susceptibility approximation:

$$
m_{\mathrm{eq}}=\frac{\mu_{0} \mu^{2} H}{3 k_{B} T}+\alpha \mu\left(\frac{\mu_{0} \mu H}{k_{B} T}\right)^{3},
$$

where the coefficient $\alpha$ (reduced third-order susceptibility) depends on the value of the dimensionless parameter $\sigma=$ $K /\left(k_{B} T\right)$ :

$$
\begin{aligned}
& \alpha=-\frac{1}{45}-8.7 \times 10^{-4} \sigma-6 \times 10^{-5} \sigma^{2} \text { if } \sigma<2, \\
& \alpha=-0.01355-0.00532 \sigma \quad \text { if } \sigma \in[2,5.5], \\
& \alpha=-\frac{1}{15}+\frac{0.13257}{\sigma}-\frac{0.0083}{\sigma^{2}} \text { if } \sigma>5.5 .
\end{aligned}
$$

Note that these approximations for $m_{\text {eq }}$ can also be used in the framework of the abrupt transition model where we have

$$
\begin{array}{ll}
\text { for } T \geqslant T_{B}: & m_{\mathrm{ZFC}}=m_{\mathrm{FC}}=m_{\mathrm{eq}}, \\
\text { for } T<T_{B}: & m_{\mathrm{ZFC}}=m_{\mathrm{b}} \text { and } \quad m_{\mathrm{FC}}=m_{\mathrm{eq}}\left(T_{B}\right) .
\end{array}
$$

*florent.tournus@univ-lyon1.fr

${ }^{1}$ D. Weller, A. Moser, L. Folks, M. E. Best, W. Lee, M. F. Toney, M. Schwickert, J. U. Thiele, and M. F. Doerner, IEEE Trans. Magn. 36, 10 (2000).

${ }^{2}$ A. Moser, K. Takano, D. T. Margulies, M. Albrecht, Y. Sonobe, Y. Ikeda, S. Sun, and E. E. Fullerton, J. Phys. D: Appl. Phys. 35, R157 (2002).

${ }^{3}$ S. Mornet, S. Vasseur, F. Grasset, and E. Duguet, J. Mater. Chem. 14, 2161 (2004).

${ }^{4}$ C. Xu and S. Sun, Polym. Int. 56, 821 (2007).

${ }^{5}$ A. H. Lu, E. L. Salabas, and F. Schüth, Angew. Chem. Int. Ed. 46, 1222 (2007).

${ }^{6}$ K. M. Krishnan, IEEE Trans. Magn. 46, 2523 (2010).

${ }^{7}$ F. Tournus and A. Tamion, J. Magn. Magn. Mater. 323, 1118 (2011).

${ }^{8}$ E. P. Wohlfarth, Phys. Lett. A 70, 489 (1979).

${ }^{9}$ R. W. Chantrell, M. El-Hilo, and K. O'Grady, IEEE. Trans. Magn. 27, 3570 (1991).

${ }^{10}$ H. Pfeiffer and R. W. Chantrell, J. Magn. Magn. Mater. 120, 203 (1993).

${ }^{11}$ M. Respaud, J. M. Broto, H. Rakoto, A. R. Fert, L. Thomas, B. Barbara, M. Verelst, E. Snoeck, P. Lecante, A. Mosset et al., Phys. Rev. B 57, 2925 (1998).

${ }^{12}$ A. Tamion, M. Hillenkamp, F. Tournus, E. Bonet, and V. Dupuis, Appl. Phys. Lett. 95, 062503 (2009).

${ }^{13}$ F. Tournus and E. Bonet, J. Magn. Magn. Mater. 323, 1109 (2011).

${ }^{14}$ M. El-Hilo, K. O’Grady, and R. W. Chantrell, J. Magn. Magn. Mater. 114, 307 (1992).

${ }^{15}$ R. Sappey, E. Vincent, N. Hadacek, F. Chaput, J. P. Boilot, and D. Zins, Phys. Rev. B 56, 14551 (1997).

${ }^{16}$ H. Kachkachi, W. T. Coffey, D. S. F. Crothers, A. Ezzir, E. C. Kennedy, M. Noguès, and E. Tronc, J. Phys.: Condens. Matter 12, 3077 (2000).

${ }^{17}$ R. K. Zheng, H. Gu, B. Xu, and X. X. Zhang, J. Phys.: Condens. Matter 18, 5905 (2006).

${ }^{18}$ The Langevin function $\mathcal{L}(x)=\operatorname{coth}(x)-1 / x$ gives the thermodynamic equilibrium response $m(H)$ for an assembly of paramagnetic spins (or macrospins, when their magnetic anisotropy can be neglected) of individual moment $\mu$ : We have $m(H)=m_{S} \mathcal{L}(\xi)$ with the dimensionless parameter $\xi=\frac{\mu_{0} \mu H}{k_{B} T}$ and $m_{S}$ as the saturation magnetic moment. For macrospins with a size distribution $\rho(V)$, we note that $\mu=M_{S} V$, where $V$ is the particle size and $M_{S}$ is the saturation magnetization, so that the total magnetic moment is obtained by integration of Langevin functions: $m=m_{S} \int \mathcal{L}_{V} \rho(V) d V$.

${ }^{19}$ Y. Shiratsuchi and M. Yamamoto, Phys. Rev. B 76, 144432 (2007).

${ }^{20}$ M. Respaud, J. Appl. Phys. 86, 556 (1999).

${ }^{21}$ H. Mamiya and I. Nakatani, IEEE Trans. Magn. 34, 1126 (1998).

${ }^{22}$ W. Wernsdorfer, E. B. Orozco, K. Hasselbach, A. Benoit, B. Barbara, N. Demoncy, A. Loiseau, H. Pascard, and D. Mailly, Phys. Rev. Lett. 78, 1791 (1997).

${ }^{23}$ M. Jamet, W. Wernsdorfer, C. Thirion, D. Mailly, V. Dupuis, P. Mélinon, and A. Pérez, Phys. Rev. Lett. 86, 4676 (2001).

${ }^{24}$ A. Tamion, E. Bonet, F. Tournus, C. Raufast, A. Hillion, O. Gaier, and V. Dupuis, Phys. Rev. B 85, 134430 (2012).

${ }^{25}$ F. Tournus, N. Blanc, A. Tamion, M. Hillenkamp, and V. Dupuis, J. Magn. Magn. Mater. 323, 1868 (2011).

${ }^{26}$ A. Hillion, A. Tamion, F. Tournus, J.-B. Flament, M. Hillenkamp, E. Bonet, and V. Dupuis, IEEE Trans. Magn. 47, 3154 (2011).

${ }^{27}$ A. Hillion, M. Pauly, A. Tamion, F. Tournus, M. Hillenkamp, B. P. Pichon, S. Begin-Colin, and V. Dupuis, J. Appl. Phys. 112, 123902 (2012).

${ }^{28}$ N. A. Usov, J. Appl. Phys. 109, 023913 (2011).

${ }^{29}$ W. F. Brown Jr., Phys. Rev. 130, 1677 (1963).

${ }^{30}$ J. L. Dormann, D. Fiorani, and M. El Yamani, Phys. Lett. A 120, 95 (1987)

${ }^{31}$ W. X. Fang, Z. H. He, D. H. Chen, and Y. Z. Shao, J. Magn. Magn. Mater. 321, 4032 (2009).

${ }^{32}$ L. E. Wenger and J. A. Mydosh, Phys. Rev. B 29, 4156 (1984).

${ }^{33}$ J. L. García-Palacios and F. J. Lázaro, Phys. Rev. B 55, 1006 (1997).

${ }^{34}$ N. A. Usov and Y. B. Grebenshchikov, J. Appl. Phys. 106, 023917 (2009).

${ }^{35}$ The linear interpolation of $\alpha(\sigma)$ between $\sigma=2$ and $\sigma=5.5$ is given by $\alpha \simeq-0.01355-0.00532 \sigma$. 
${ }^{36}$ A. Perez, P. Melinon, V. Dupuis, L. Bardotti, B. Masenelli, F. Tournus, B. Prevel, J. Tuaillon-Combes, E. Bernstein, A. Tamion, N. Blanc, D. Tainoff, O. Boisron, G. Guiraud, M. Broyer, M. Pellarin, N. Del Fatti, F. Vallee, E. Cottancin, J. Lerme, J-L. Vialle, C. Bonnet, P. Maioli, A. Crut,
C. Clavier, J. L. Rousset, F. Morfin, Int. J. Nanotech. 7, 523 (2010).

${ }^{37}$ A. Tamion, C. Raufast, M. Hillenkamp, E. Bonet, J. Jouanguy, B. Canut, E. Bernstein, O. Boisron, W. Wernsdorfer, and V. Dupuis, Phys. Rev. B 81, 144403 (2010). 